\title{
Enhanced Downlink Capacity in UMTS Supported by Direct Mobile-to-Mobile Data Transfer
}

\author{
Larissa Popova, Thomas Herpel, and Wolfgang Koch \\ University Erlangen-Nuremberg, Germany \\ \{popova,koch\}@LNT.de \\ Thomas.herpel@freenet.de
}

\begin{abstract}
The goal of this work is first to analyze the feasibility of a peer-to-peer file sharing technique in mobile cellular environments, taking into account key characteristics and peculiarities of the UMTS Radio Access Network (UTRAN). The concept is referred here to as mobile-tomobile $(\mathrm{m} 2 \mathrm{~m})$. Next, our research efforts explore the performance benefits of $\mathrm{m} 2 \mathrm{~m}$ file sharing applications in UMTS networks in terms of releasing overall downlink capacity, which can be used to provide better Quality of Service (QoS) for real-time services. To evaluate the performance of the proposed $\mathrm{m} 2 \mathrm{~m}$ concept we conducted extensive simulation studies with appropriately modified radio propagation models for low antenna heights for both, transmitter and receiver, as it is typical for $\mathrm{m} 2 \mathrm{~m}$. Two alternative scenarios of serving user requests $(\mathrm{m} 2 \mathrm{~m}$ network mode and conventional UMTS mode) have been constructed and analyzed. The results indicate a dramatic increase in service probability and overall throughput gain of up to $85 \%$ in a UMTS network, supported by the $\mathrm{m} 2 \mathrm{~m}$ data transmission mode. Furthermore, results show that by a well-designed $\mathrm{m} 2 \mathrm{~m}$ routing policy and proper utilization of currently not used uplink resources (due to asymmetric uplink/downlink traffic load) substantial reduction of the expected file download time can be achieved.
\end{abstract}

Keywords: Direct mobile-to-mobile data transfer, WCDMA, released downlink capacity, asymmetric uplink/downlink traffic load, unified radio interface, data exchange policy.

\section{Introduction}

Rapid growth of new multimedia services such as real-time streaming, distributed video conferences, with their demand for high data rates, as well as downloading of popular movies or music files put a considerable load onto the valuable and limited resources of radio networks. It is well known that in UMTS networks the traffic load is asymmetrically distributed between uplink/downlink. Typically, the downlink is the potential bottleneck, while free resources may be available in the uplink. Thus, a proper management of the air interface resources is a challenging task in order to utilize the spectral resources more efficiently. 
Nowdays, mobile networks are widely used for so-called background services such as digital camera images, mp3 file downloads or new movie trailers. Although these services are interesting for many users, conventional solutions for capacity utilization such as multicast or broadcast are not applicable since users request service at different times. The above considerations stimulate research for employing new paradigms in the architecture of cellular communication systems, as well as in policies in handling of user service requests.

In this work we propose an approach to overcome the capacity limitations of cellular networks and to prevent exhaustion of the downlink capacity by enabling direct data transmission among the background users within a radio network.

Currently, there are only a few proposals for a mobile peer-to-peer file sharing architecture. In [7] the authors are motivated by the potential UMTS capacity improvement of embedding WLAN (Wireless Local Area Network) systems in UMTS. The effort of this research is focused on the basic applicability and enhanced routing of the information in the proposed hybrid network by using a series of short mobile-to-mobile hops between already connected mobiles to extend coverage into areas not covered by the conventional UMTS cell. The propagation model they used is the free-space model, which is not realistic for terrestrial scenarios. The feasibility of the eDonkey Internet protocol in a GPRS environment was investigated in [46] and extended to UMTS radio networks in [5]. The main focus lies on resource mediation and control by using different strategies for data caching in the wired part of the network. Thus, signalling and data traffic is displaced from the air interface to the core network. No direct data transmission among the users is intended.

All the above mentioned studies are based on entirely different approaches and have other goals than the concept, proposed in this paper.

In our concept the users that are interested in downloading a popular file form a mobile cooperative community (groups) and using the fact that traffic load of multimedia services is asymmetrically distributed between uplink and downlink, contribute their own currently not used uplink capacity for providing the packets of the content to other users in the group (in their coverage range) in multicast mode on the uplink carrier frequencies. Our concept is denoted as $\mathrm{m} 2 \mathrm{~m}$ (mobileto-mobile). Although we focus on the FDD (Frequency Division Duplex) mode of WCDMA (Wideband Code Division Multiple Access) the principle can be applied to other systems as well.

Particularly, in hotspot environment users increasingly demand ubiquitous data availability. Thus, the main focus of our analysis lies in the optimization of data availability to users in hotspots (e.g. airports, railway stations) by using the $\mathrm{m} 2 \mathrm{~m}$ file sharing technique. We consider the background user population, dynamically joining and leaving the system. The users organize communities which are interested, for example, in the movie trailer or the latest computer games. Groups are dynamically reshaped, so that the members of each group represent a relatively loosely coupled formation. Instead of transmission of the complete data file by providing individual links from the Node B to each user, the original file, which is available somewhere in the network, is divided into 
$m$ logical packets and distributed packet by packet to active background users in order to generate one complete copy of this file in every radio cell. A user which received a packet from Node B acts as a server for that particular packet. The mobile terminals (MTs) are assumed to be able to receive in both uplink and downlink and each new user brings further uplink resources into the system. By this cooperative data transfer among the users, the Node B does not need to spend a lot of its valuable resources to accommodate the population of the above mentioned background user-class, as a result a major part of the traffic is shifted away from the downlink, making the released downlink capacity available for other (e.g. real-time demanding) services.

The main goal of this work is to show the feasibility of the proposed $\mathrm{m} 2 \mathrm{~m}$ technique for cellular radio networks like UMTS and to analyze advantage of using $\mathrm{m} 2 \mathrm{~m}$ application in some specific wireless scenarios, which, we believe, are of practical importance.

The performance results were evaluated taking into account the specifics of UTRAN, e.g. wireless interference, realistic propagation model, etc.

\section{M2M Model Characteristics and Assumptions}

\subsection{M2M Concept}

To enable the wireless peer-to-peer technique we revised the idea of a mesh cooperative architecture for the fixed-line Internet [2] and appropriately extended it for the UTRA-FDD (Frequency Division Duplex).

In Figure 1 the main concept of the $\mathrm{m} 2 \mathrm{~m}$ technique is demonstrated. MTs, which are interested in downloading a popular content participate in the file sharing via direct $\mathrm{m} 2 \mathrm{~m}$ data transfer with the purpose to reconstruct this original popular content, which is distributed in the network. However, in contrast to Internet peer-to-peer applications in a cellular system the number of users who can cooperate with each other is limited by the transmit power of the MT and its coverage range, which will be typically less than a cell. Therefore, in case of wireless cooperative community formation, mobile communities are location and radio propagation dependent. Thus, to cooperate with each other, $\mathrm{m} 2 \mathrm{~m}$ users must be organized into groups with nearby located users. Grouping is not restricted to users of one cell. The decision to which group a user should be assigned is based strictly on current propagation conditions, described in Section III. Upon arrival each new user, which is interested in download of the popular content, establishes contact to the Node B/RNC (Radio Network Controller) in order to get an authorization to participate in $\mathrm{m} 2 \mathrm{~m}$ file transfer and to get information about nearby located $\mathrm{m} 2 \mathrm{~m}$ users. All authorized $\mathrm{m} 2 \mathrm{~m}$ users must allow using of their uplink capacity for providing the packets of the content, he has requested, to other $\mathrm{m} 2 \mathrm{~m}$ users, which are interested in it. In order to reduce the transmission of identical packets on the network links the $\mathrm{m} 2 \mathrm{~m}$ transfer is performed in multicast mode on the normally only partly used uplink carrier frequency, whereas receivers in the group switch to listen on the uplink. 


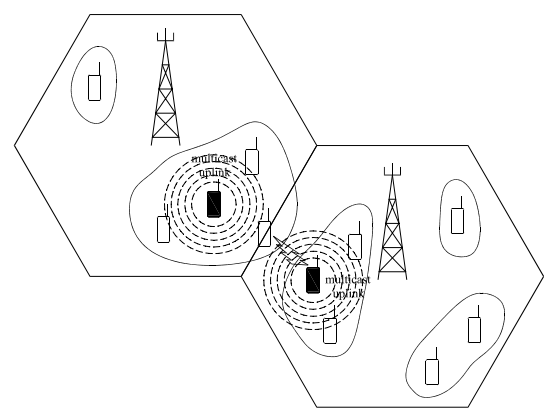

Fig. 1. M2M Concept

\subsection{Radio Interface Restrictions}

In the following some restrictions and potential solutions of implementing a peerto-peer technique in UMTS Radio Access Network (UTRAN) are listed.

- One of the main restrictions of UTRAN is wireless interference. In order to avoid interference from an MT transmitting in $\mathrm{m} 2 \mathrm{~m}$ mode on other signals at the Node B receiver, the transmit power is set to the minimum, which is $-44 \mathrm{dBm}$ according to $3 \mathrm{GPP}$ specifications [1].

- Compared to fixed-line networks the air interface of wireless systems has a relatively limited transmission capacity. Furthermore, the effect of user blocking, as a consequence of congestion control, is expected to occur more often than in fixed-line environments in both directions. Thus, in order to increase the efficiency of uplink bandwidth usage it is necessary to maximally reduce the signalling information. This can be done by taking advantage of already existing infrastructure of mobile communication systems, e.g. the network providers know the online status and service agreement of the mobile users.

- The limitation of battery capacity of the handsets results in a lower online time compared to the fixed-line desktop PCs. Thus, a well designed file sharing organization between mobiles is essential.

- There is a need for appropriate propagation models for wireless peer-to-peer links.

The problems and challenges such as a fair scheduling, battery life, billing, security and rights management could be mitigated by strategies such as 1) offering bonus system (e.g. upload credits) to network subscribers who choose to allow using their uplink capacity to provide the contents to other users, 2) by implementing restrictions on the amount of traffic that a particular terminal is permitted to distribute, to save the battery life. The contents liable to cost can be also distributed with $\mathrm{m} 2 \mathrm{~m}$ strategy, might be, however, protected, for example, via DRM (Digital Rights Management). However, all analysis in this work are mainly focused on the technological characteristics of mobile peer-to-peer applications and the proper consideration of above mentioned issues is not a topic of this paper. 


\subsection{Traffic Model}

We study the performance of the proposed $\mathrm{m} 2 \mathrm{~m}$ concept in a UMTS network with dynamic user arrival and departure pattern. User arrivals are modelled by a Poisson process and the MTs are randomly distributed over the cell area. All users are assumed to be pedestrians. We assume that there are mobile specific content types, like $\mathrm{mp} 3$, to be distributed with $\mathrm{m} 2 \mathrm{~m}$ strategy. The sizes of popular mp3 files used in our simulation have been taken from [4].

\subsection{M2M Propagation Model}

Successful data transmission in wireless systems depends on radio propagation conditions. The characteristic of the $\mathrm{m} 2 \mathrm{~m}$ channels is significantly different from conventional Node B - MT links because of the low antenna heights of both receiver/transmitter and a relatively short propagation distance.

Pathloss and Shadowing: In our work the shadowing process is characterized by a lognormal distribution with standard deviation $\sigma=6 \mathrm{~dB}$ and the pathloss between two MTs is calculated using extended Okumura-Hata model [3]. Although, the application of this model is generally restricted to antenna heights of Node B starting from $30 \mathrm{~m}$, the direct comparison of this model with a model from 8] tailored for low antenna heights at both sides shows a good suitability of the model of 3 for the present application. Figure 2 shows a scatter plot for the received power depending on transmitter/receiver separation (a minimum transmit power of $-44 \mathrm{dBm}$ was assumed).

Fading: For the simulated traffic model, cf. Subsection $C$, flat small-scale fading (constant within one radio frame $(0.01 \mathrm{sec})$ ) is assumed for the $\mathrm{m} 2 \mathrm{~m}$ links.

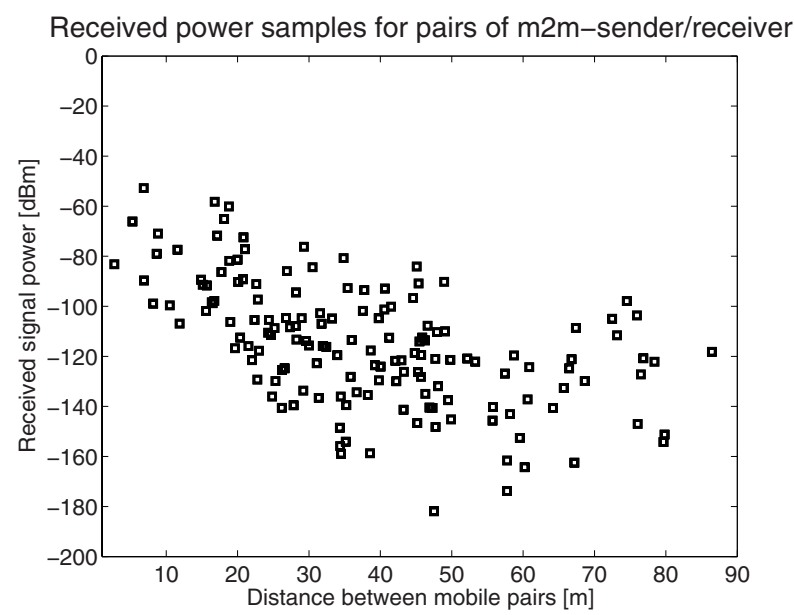

Fig. 2. Scatter plot of received signal power for $\mathrm{m} 2 \mathrm{~m}$ links as a function of pathloss and shadowing, constant transmit power of $-44 \mathrm{~dB}$ 


\section{Algorithm}

We simulate a population of users that are interested in downloading a popular file, which originally is only available in the core network. Since downlink capacity is limited, Node B does not serve all users simultaneously, and the popular file is divided into $m$ logical packets, each with an individual ID, and distributed periodically (every 10 frames) packet by packet to active background users in order to generate one complete copy of this file in every radio cell. A user which received a packet from the Node B behaves as a server for that particular packet and uses its uplink resources for providing the packet. The users are organized into dynamically reshaped multiple groups to cooperate with each other with the purpose to reconstruct the original file.

\subsection{Group Organization Policy}

In the following general assumptions for the organization of dynamic groups in $\mathrm{m} 2 \mathrm{~m}$ file sharing are presented.

- Each new user looks for a group to join when trying to receive popular content.

In case that no appropriate group for a new $\mathrm{m} 2 \mathrm{~m}$ user is found he forms a group with stand-alone user only and waits until the next group update.

- MTs form groups, which satisfy the following conditions:

$$
\begin{array}{r}
10 \lg P_{T X}-L_{i j}-\Lambda_{i j} \geq-112 \mathrm{dBm} \mid \\
P_{T X}=P_{T X, \text { min }}, \forall i, j \in \text { group },
\end{array}
$$

where $L_{i j}$ is the pathloss between MTs $i$ and $j$ and $\Lambda_{i j}$ is the random variable describing the shadowing process.

- The simplest way to inform a new MT about all other MTs, already requesting the same content in its coverage range is to transmit "Hello" packets by all MTs, periodically. But this procedure puts considerable load on signalling channels. Thus, it would be more efficient if upon arrival each new $\mathrm{m} 2 \mathrm{~m}$ user contacts a Node B that provides information about all other $\mathrm{m} 2 \mathrm{~m}$ users already in the system in the range of tens of meters, to determine the potential members of the group (triangulation or GPS). Only MT, assigned to a multiple member group sends a "Hello" packet to get an appropriate information about the pathloss to any other MTs from its group. In such a way we can reduce the signalling information between stand-alone MTs and prolong the limited battery life of MTs.

- The size of the groups is restricted to a maximum number of members.

- Users in the group dynamically join and leave the group at any time (battery life, handover). Each MT can be a member of only one group at the same time. Groups are periodically updated (every 100 radio frames) and reshaped in order to check positions of MTs and their radio propagation characteristics on the one hand, and to track and authorize new $\mathrm{m} 2 \mathrm{~m}$ users or handover-users in the group in case they fulfill the above mentioned "joingroup" criteria, on the other hand. 
- Information about the link quality can be obtained e.g. from "Hello" packets, periodically transmitted by MTs.

- Grouping is not restricted to one cell.

\subsection{Data Exchange Policy}

Using the $\mathrm{m} 2 \mathrm{~m}$ service, the MT concurs in the employment of its own free uplink resources (service level agreement). We assume that each user knows about the packets it has downloaded and the packet IDs that are available at its neighbors. The data exchange algorithm finds an appropriate "sender" candidate, based on a local "most-utile-packet" scheme, in order to maximize the number of users for which the packet can be useful. This procedure is performed framewise. After the best candidate is found, the admission control procedure verifies whether the system has enough uplink capacity to accept the connection. If there is more than one sender candidate or if a sender candidate has more than one packet to send, the packet with the lowest ID will be distributed.

In order to avoid packet collisions caused by wireless interference from other groups, to reduce identical packets on the network links and in turn to utilize uplink bandwidth more efficiently, our concept uses the so-called multicast mode among users within a group. Such a parallel packet downloading policy improves the performance of the system, in terms of number of simultaneously served $\mathrm{m} 2 \mathrm{~m}$ users. Users, which have not found any useful packets within a specified time interval, try to connect to the Node B for packet delivery 1 We now summarize the main characteristics/assumptions of our data exchange policy:

- Initially, no packet is available among m2m users.

- The data exchange policy is based on a local "most-utile-packet" scheme.

- Packets can be distributed in an arbitrary order.

- The $\mathrm{m} 2 \mathrm{~m}$ data transfer is performed in multicast mode on the uplink carrier frequency; identification of the sender is done using a unique scrambling code.

- The MTs must be able to receive in both uplink and downlink.

- No physical data channel on Node B is needed to control the intra-group data transfer.

- The Node B/RNC responsibilities are 1) to distribute at least one complete copy of the original file in every radio cell (time interval between Node B "packets upload sessions" is 10 radio frames), 2) to support the data exchange process in the $\mathrm{m} 2 \mathrm{~m}$ groups with signalling information such as "listen on the uplink frequency", etc. 3) to serve timeout requests from MTs.

On the one hand the last point of the download policy gives the MTs better chances of finding missing packets and finishing the download faster, on the other hand, however, it puts additional load on the downlink resources. To more or less overcome this problem and to enable a more efficient data exchange by minimizing the probability of serving $\mathrm{m} 2 \mathrm{~m}$ requests via Node $\mathrm{B}$, it is necessary to estimate the optimal group size.

\footnotetext{
${ }^{1}$ The MT can receive the single packets of a data file in a random order.
} 


\section{Performance Evaluation}

In this section we evaluate the performance of the proposed $\mathrm{m} 2 \mathrm{~m}$ concept through simulations.

We first list the assumptions and parameter settings we employed in our simulations:

- The MTs are distributed randomly over the cell area. The radius of the cell is $50 \mathrm{~m}$ (hotspot scenario).

- The MTs generate a Poisson arrival process with rate $\lambda$ of requests for some popular content.

- The maximum group size varies between 3-10.

- Users within the group dynamically join and leave the group at any time (handover, battery discharging).

- The MTs depart from the system immediately after finishing their download.

- The size of a logical packet is equal to one UMTS radio frame 2

- The quality of the wireless channel in each group remains constant within each radio frame, but can vary from frame to frame (block-fading channel).

- If the packet is incorrect after detection, we declare a packet loss.

- The simulation time is 400 - $1200 \mathrm{sec}$ and we collect data framewise.

\subsection{Comparison of M2M with Conventional File Sharing}

First, we focus on the download data volume characteristics when only one popular file is dispersed with the $\mathrm{m} 2 \mathrm{~m}$ technique. We compare performance results with those for the conventional UMTS mode, where a continuous transmission of data is organized by individual links from Node B to each user.

We consider three performance measures:

- Overall downlink throughput gain: data volume reduction in the downlink.

- Service probability gain: number of served users.

- Download time gain: download time reduction. We define the download time as the time window, in which the user receives the complete file. The criterion for the download time gain is the $90 \%$ quantile of finished downloads in the system.

The most important simulation parameters are summarized in Table 1

Figure 3 shows the system performance versus the offered traffic load.

The upper graphs demonstrate the efficiency of the $\mathrm{m} 2 \mathrm{~m}$ file sharing mechanism for medium and heavy traffic load in terms of the download time reduction. The bottom graphs depict the $\mathrm{m} 2 \mathrm{~m}$ performance gain in terms of released downlink resources.

As one would expect, the results show that the higher the traffic load, the more efficient is the performance of $\mathrm{m} 2 \mathrm{~m}$ file sharing. Obviously, with increased traffic load $\mathrm{m} 2 \mathrm{~m}$ users have better opportunities to find the content of interest, which

${ }^{2}$ Depending on the coding scheme and spreading factor this leads to corresponding packet sizes. 
Table 1. Main simulation parameters

\begin{tabular}{|l|l|}
\hline Traffic and environmental settings & \\
\hline Traffic load (max. number of $\mathrm{m} 2 \mathrm{~m}$ users/cell) & 10 (low), 30 (medium), 50 (high) \\
\hline Maximum $\mathrm{m} 2 \mathrm{~m}$ group size & $3,7,10$ \\
\hline Antenna type & omni-directional \\
\hline Cell radius & $50 \mathrm{~m}$ \\
\hline Moving process for MT & Gaussian Random Walk \\
\hline User profile & Pedestrian \\
\hline Radio interface and algorithmic settings & \\
\hline File size & $500 \mathrm{KByte}$ \\
\hline Required data rate & $60 \mathrm{~kb} / \mathrm{s}$ \\
\hline Maximum user data rate with $1 / 2-$ rate coding & $30 \mathrm{~kb} / \mathrm{s}$ \\
\hline Size of logical packets for $\mathrm{m} 2 \mathrm{~m}$ data & $225 \mathrm{bit}(\mathrm{coded})$ \\
\hline Receiver sensitivity & $-112 \mathrm{dBm}$ \\
\hline Transmission power in $\mathrm{m} 2 \mathrm{~m}$ mode & $-44 \mathrm{dBm}$ \\
\hline$E_{b} / N_{0}$ target & $3 \mathrm{~dB}$ \\
\hline Inner loop power control for m2m sender & OFF \\
\hline Simulation step & $1 \mathrm{radio}$ frame $(0.01 \mathrm{sec})$ \\
\hline Group update period & $100 \mathrm{radio}$ frames $(1 \mathrm{sec})$ \\
\hline
\end{tabular}

positively influences the file downloading time, on the one hand and increases downlink throughput capacity on the other hand, making the released Node B capacity available to other services.

Table 2 shows some numerical values for the overall downlink throughput gain and relative download time reduction for complete file download in $\mathrm{m} 2 \mathrm{~m}$ network mode compared with conventional UMTS data transmission (+: $\mathrm{m} 2 \mathrm{~m}$ slower, - : $\mathrm{m} 2 \mathrm{~m}$ faster). The rows "Data volume in DL in conv. mode [MB/cell]" and "Data volume in DL in m2m [MB/cell]" show how many Mbyte of data had to be sent via the downlink channels in order to distribute the data file of 500 Kbyte size to the users within one cell. We examine now some parameters and their influence on the system performance.

\subsection{Impact of Group Size}

We consider again the same $\mathrm{m} 2 \mathrm{~m}$ scenario with maximum group size $3,7,10$ and investigate the effect of the group size on the performance of the proposed

Table 2. Overall downlink throughput gain

\begin{tabular}{|l|c|c|r|}
\hline Load & low & medium & high \\
\hline Data volume in DL in conventional mode [MB/cell] & 5.73 & 16.51 & 34.44 \\
\hline Data volume in DL in m2m mode [MB/cell] & 1.33 & 2.93 & 4.67 \\
\hline Released DL capacity [\%] & 76.70 & 82.23 & 86.44 \\
\hline Time m2m [sec] & +189.3 & -20.1 & -142.3 \\
\hline
\end{tabular}



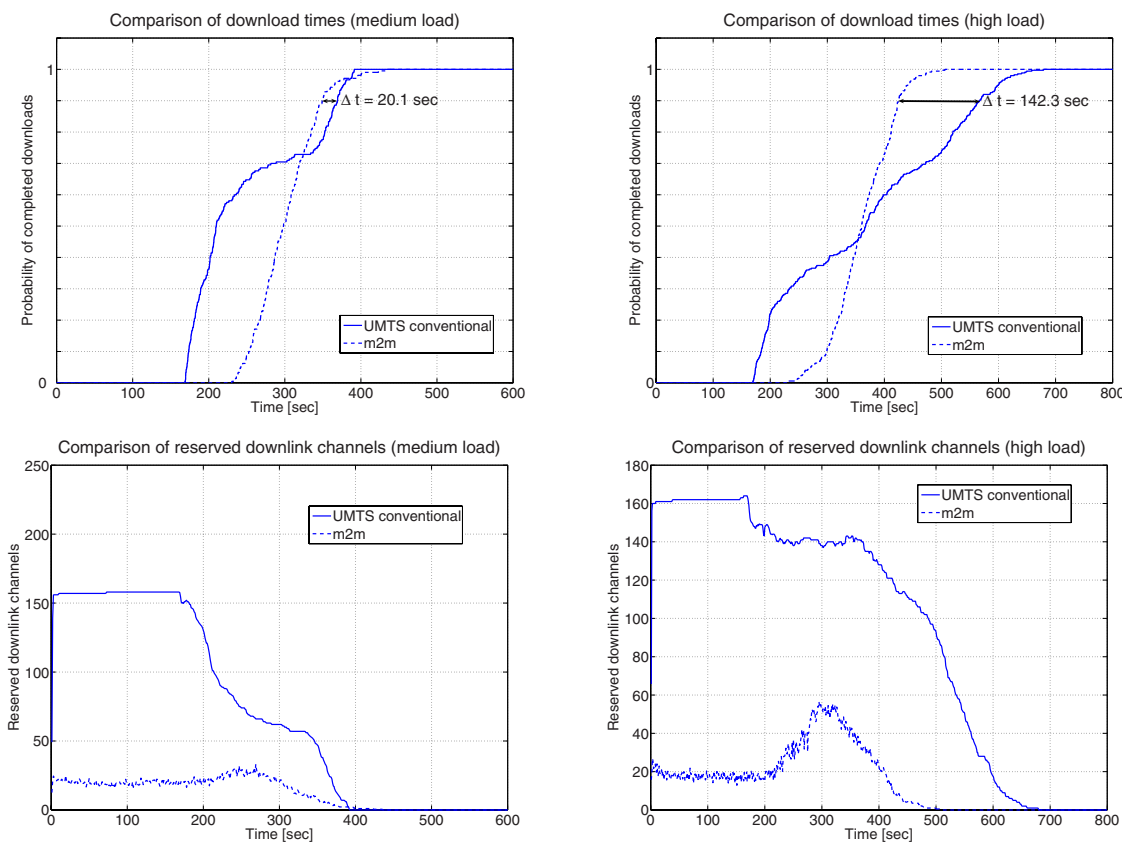

Fig. 3. Performance comparison of conventional and $\mathrm{m} 2 \mathrm{~m}$ mode for medium traffic load (left) and high traffic load (right), group size $=7$

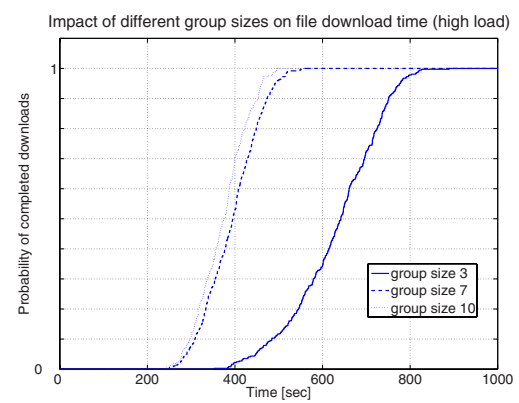

Fig. 4. Impact of the group size on the file download time for high traffic load

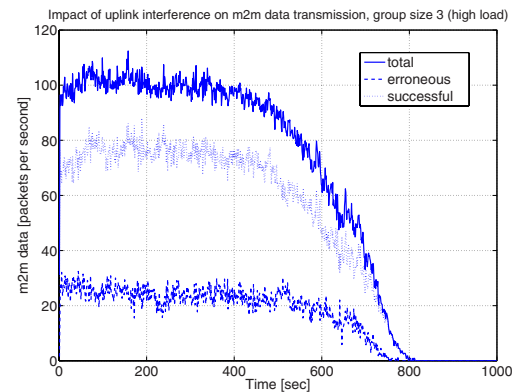

Fig. 5. Impact of uplink interference on $\mathrm{m} 2 \mathrm{~m}$ data transmission for group sizes 3 in a high loaded UMTS system

technique for low, medium and high traffic load. In figure 4 the influence of the group size on the file download time for a high loaded UMTS system is illustrated.

Intuitively, the larger the group size, the higher the multicast efficiency. The number of needed senders is lower, hence, for the same number of members in 
Table 3. Impact of uplink interference for different group sizes and traffic scenarios (erroneous data [\%])

\begin{tabular}{|c|c|c|c|}
\hline \multirow{2}{*}{ Different System Scenarios } & \multicolumn{3}{|c|}{ Erroneous data, \% } \\
\cline { 2 - 4 } & group size 3 & group size 7 & group size 10 \\
\hline low load & 3.71 & 1.86 & 2.07 \\
\hline medium load & 13.70 & 5.56 & 6.25 \\
\hline high load & 18.96 & 8.88 & 8.99 \\
\hline
\end{tabular}

the system large groups consume less bandwidth. Equivalently, with the same uplink resource consumption more members will get a service when the group size is bigger.

With increased number of multiple coexisting groups (group size 3 ) in the network some performance degradation of the $\mathrm{m} 2 \mathrm{~m}$ technique was observed. This effect is influenced by admission control, wireless interference and user mobility. Obviously, with increasing the number of groups the number of sender candidates that can be admitted is bounded by the uplink capacity. It results in a rejection of some link admission requests of sender candidates. Besides, if the group size is too small and the mobility of users is low (as assumed in this work), the probability to find in each frame a missing packet is quite low; the number of packet requests from $\mathrm{m} 2 \mathrm{~m}$ users to Node $\mathrm{B}$ increases and puts additional load on the downlink resources.

Another important performance criterion is the average rate of the successfully delivered packets. For systems with small group size we observed enormous m $2 \mathrm{~m}$ uplink interference, which leads to significant performance degradation and in turn to an increase of download time.

Figures 5 and 6 demonstrate the impact of the uplink interference on $\mathrm{m} 2 \mathrm{~m}$ data transmission for group sizes 3 and 7 in a high loaded UMTS system (effective user data rate is $30 \mathrm{~kb} / \mathrm{s}$ ). Relative losses of link quality (number of corrupted data in \%) for different group sizes and traffic scenarios are shown in Table 3 .

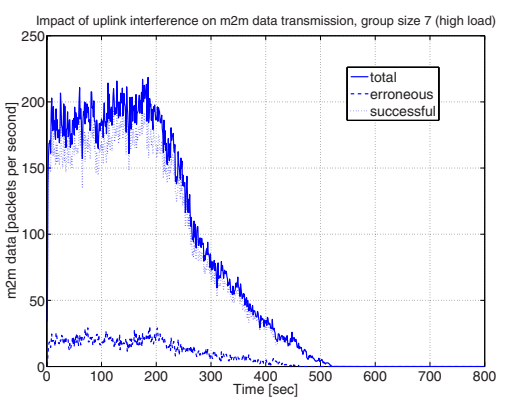

Fig. 6. Impact of uplink interference on $\mathrm{m} 2 \mathrm{~m}$ data transmission for group sizes 7 in a high loaded UMTS system

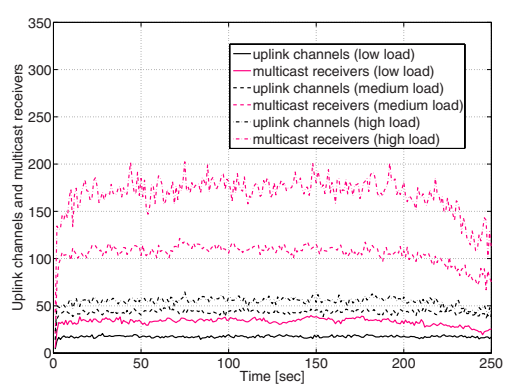

Fig. 7. Instantaneous number of transmitting MTs and corresponding number of receiving multicast MTs, respectively, in the cluster of 7 cells 
Table 4. The average number of multicast receivers per group for different $\mathrm{m} 2 \mathrm{~m}$ traffic scenarios

\begin{tabular}{|l|c|c|}
\hline Load & Users per one UL-channel & Service probability gain, \% \\
\hline $\mathrm{m} 2 \mathrm{~m}$ (low) & 1.91 & 46.92 \\
\hline $\mathrm{m} 2 \mathrm{~m}$ (medium) & 2.43 & 58.40 \\
\hline $\mathrm{m} 2 \mathrm{~m}$ (high) & 3.11 & 67.30 \\
\hline
\end{tabular}

\subsection{Impact of Multicast Technique}

As the required data rate for each $\mathrm{m} 2 \mathrm{~m}$ request is always the same it is correct to evaluate the effectiveness of the proposed concept with respect to the relative service probability gain by using our knowledge about the number of successfully delivered packets in each multicast group every frame.

Figure 7 and Table 4 demonstrate the benefit of using multicast in terms of the relative gain in the number of MTs, that can be supported by $\mathrm{m} 2 \mathrm{~m}$ concept.

Comparing our performance results with those of the conventional UMTS mode, one can observe a dramatic increase in service probability by using the proposed $\mathrm{m} 2 \mathrm{~m}$ technique.

\section{Conclusions and Perspectives}

In this paper, a concept based on direct $\mathrm{m} 2 \mathrm{~m}$ data exchange on uplink channels is presented and its performance for UMTS is analyzed. Our concept targets the distribution of large files in a dynamic wireless environment, where multiple user groups coexist in the UMTS network by taking wireless interference among the neighboring groups into account. The main focus of our analysis lies in the optimization of data availability to users in hotspots (e.g. airports, railway stations), since in hotspot environment users increasingly demand ubiquitous data availability. This technique does not require any centralized knowledge of the transfers in the rest of the network to perform the intra-group data transfer, but requires cooperative MTs. The flexible nature of the cooperative community formation makes the proposed algorithm robust to sudden departure of any member in the group. Furthermore, we demonstrated through extensive simulations the performance advantages of using $\mathrm{m} 2 \mathrm{~m}$ mode in UMTS network for distribution of popular non-real time contents compared to the conventional UMTS mode. The following conclusions can be drawn from the numerical results:

- The higher the arrival intensity and mobility of $\mathrm{m} 2 \mathrm{~m}$ users, the more bandwidth becomes available. As a consequence, the more efficient is the $\mathrm{m} 2 \mathrm{~m}$ file sharing.

- The performance benefits provided by the $\mathrm{m} 2 \mathrm{~m}$ technique in terms of released downlink capacity are up to $85 \%$ compared to the conventional UMTS mode.

- Furthermore, we observe up to $21 \%$ of download time reduction for complete file download in a UMTS network, supported by $\mathrm{m} 2 \mathrm{~m}$ data transmission mode. 
- Besides, the number of MTs that can be supported by such a system is more than three times higher than in conventional UMTS.

In this work only a few examples of numerical results are shown. In our studies we have conducted many more simulations, than can be presented here with different parameter settings to investigate the feasibility of our concept. Our purpose was to demonstrate in this paper the advantage of using $\mathrm{m} 2 \mathrm{~m}$ application in some specific wireless scenarios, which, we believe, are of practical importance. According to the achieved results, the proposed algorithm might be a promising alternative for distribution of popular content in cellular radio networks like UMTS and motivate further analysis of using $\mathrm{m} 2 \mathrm{~m}$ technique for a wide range of scenarios.

\section{References}

1. UE Radio Transmission and Reception. 3GPP TS 25.101.

2. B. Cohen. Incentives Build Robustness in BitTorrent. In Workshop on Economics of Peer-to-Peer Systems, Berkeley, CA, USA, May 2003.

3. E. Damosso. Digital Mobile Radio towards future generation systems, COST Report 231. In Proc. of IEEE PIMRC, Taipei, Taiwan, October 1996.

4. T. Hossfeld, K. Tutschku, and F. Andersen. Mapping of File-Sharing onto Mobile Environments: Feasibility and Performance of eDonkey with GPRS. In Proc. of IEEE WCNC, New Orleans, USA, March 2005.

5. T. Hossfeld, K. Tutschku, and F. Andersen. Mapping of Filesharing onto Mobile Environments: Enhancement by UMTS. In Proc. of IEEE Pervasive Computing and Communications (PerCom), Kauai Island, Hawaii, March 2005.

6. T. Hossfeld, K. Tutschku, F. Andersen, H. de Meer, and J. Oberender. Simulative Performance Evaluation of a Mobile Peer-to-Peer File-Sharing System. In Proc. of IEEE Next Generation Internet Networks (NGI), Rome, Italy, April 2005.

7. D. Tacconi, C. Saraydar, and S. Tekinay. Ad Hoc Enhanced Routing in UMTS for Increased Packet Delivery Rates. In Proc. of IEEE WCNC, New Orleans, USA, March 2005.

8. Z. Wang, E. Tameh, and A. Nix. Statistical Peer-to-Peer Channel Models for Outdoor Urban Environments at $2 \mathrm{GHz}$ and $5 \mathrm{GHz}$. In Proc. of IEEE VTC Fall, Los Angeles, USA, September 2004. 